\title{
Local absorption spectra of artificial atoms and molecules
}

\author{
C. D. Simserides, U. Hohenester, G. Goldoni, and E. Molinari \\ Istituto Nazionale per la Fisica della Materia (INFM) and Dipartimento di Fisica, \\ Università di Modena e Reggio Emilia, Via Campi 213A, I-41100 Modena, Italy
}

\begin{abstract}
We investigate theoretically the spatial dependence of the linear absorption spectra of single and coupled semiconductor quantum dots, where the strong three-dimensional quantum confinement leads to an overall enhancement of Coulomb interaction and, in turn, to a pronounced renormalization of the excitonic properties. We show that - because of such Coulomb correlations and the spatial interference of the exciton wavefunctions - unexpected spectral features appear whose intensity depends on spatial resolution in a highly non-monotonic way when the spatial resolution is comparable with the excitonic Bohr radius. We finally discuss how the optical near-field properties of double quantum dots are affected by their coupling.
\end{abstract}

\section{INTRODUCTION}

In recent years much attention is being devoted to the properties of semiconductor quantum dots (QDs). In these systems, carriers are subject to a confining potential in all spatial directions, giving rise to a discrete energy spectrum ("artificial atoms") and to novel phenomena of interest for fundamental physics as well as for applications to electronic and optoelectronic devices [1.2]. The extension and the shape of the QD confining potential varies, depending on the nanostructure fabrication technique: The dots that are studied most extensively by optical methods are induced by quantum well (QW) thickness fluctuations [3 6 ], or obtained by spontaneous island formation in strained layer epitaxy [7 9], self-organized growth on patterned substrates [10], stressor-induced QW potential modulation [1], cleaved edge overgrowth 12], as well as chemical self-aggregation techniques [13,14]. The resulting confinement lengths fall in a wide range between $1 \mu \mathrm{m}$ and $10 \mathrm{~nm}$.

In spite of the continuing progress, all the available fabrication approaches still suffer from the effects of inhomogeneity and dispersion in the dot size, which lead to large linewidths when optical experiments are performed on large QD ensembles. A major advancement in the field has come from different types of local optical experiments, that allow the investigation of individual quantum dots thus avoiding inhomogeneous broadening [3 14].

Among local spectroscopies, the approaches based on scanning near-field optical microscopy (SNOM) 15] are especially interesting as they bring the spatial resolution well below the diffraction limit of light: With the development of small-aperture optical fiber probes, subwavelength resolutions were achieved $(\lambda / 8-\lambda / 5$ [16] or $\lambda / 40$ [17) and the first applications to nanostructures became possible [5, 6, 18 23]. As the resolution increases, local optical techniques in principle allow direct access to the space and energy distribution of quantum states within the dot. This opens, however, a number of questions regarding the interpretation of these experiments, that were often neglected in the past.

First of all, for spatially inhomogeneous electromagnetic (EM) fields it is no longer possible to define and measure an absorption coefficient that locally relates the absorbed power density with the light intensity (since the susceptibility $\chi\left(\mathbf{r}, \mathbf{r}^{\prime}\right)$ cannot be approximated by a local tensor). In the linear regime, a local absorption coefficient can still be defined, which is however a complicated function that depends on the specific EM field distribution [24]. The interpretation of near-field spectra therefore requires calculations based on a reasonable assumption for the profile of the EM field.

Secondly, the quantum states that are actually probed are few-particle states of the interacting electrons and holes photoexcited in the dot. Even in the linear regime, excitonic effects are known to dominate the optical spectra of dots since Coulomb interactions are strongly enhanced by the three-dimensional confinement. Near-field spectra probe exciton wavefunctions, and their spatial coherence and overlap with the em-field profile will determine the local absorption 24].

In this paper, we show how the above phenomena affect local spectra of QDs, with special attention to the case of coupled dots ("artificial molecules") where carriers interact across the barrier via tunneling and/or Coulomb matrix elements [25]. Indeed, the optical properties of coupled dots are currently of great interest not only in view of the unavoidable inter-dot interactions occurring in real samples with dense QD packing, but also in view of their relevance for designing novel devices including gates for possible solid-state implementations of quantum information processing [26].

We will show that the relative phase of the exciton wavefunction in adjacent coupled dots (or in different regions of the same dot) can induce dramatic changes in the selection rules with respect to far-field spectra: A realistic prediction of these effects require accurate calculations taking into account quantum confinement as well as Coulomb interactions. Our theoretical scheme is especially designed to allow a realistic description of 
the quantum states of the interacting electron and holes photoexcited in the linear regime. In this respect we improve drastically over previous approaches, which generally focused on a more detailed treatment of the EM field distributions [27 31 .

Our theoretical framework for dots is summarized in Section III, while Sections III and IV] discuss our results and conclusions for single and coupled dots.

\section{THEORY}

In this section, we summarize our theoretical approach for computing local absorption spectra for semiconductor QDs. We first show in Sec. II A how to compute the single-particle eigenstates for electrons and holes subjected to a three-dimensional confinement potential. These single-particle states are then used in Sec. II B for the calculation of electron-hole (i.e., optical) excitations. In analogy to semiconductor systems of higher dimensionality, we shall refer to these excitations as excitons; the properties of such excitons, however, are not only governed by the attractive electron-hole Coulomb interaction but in addition by the strong quantum confinement. Finally, we use in Sec. II C the above ingredients to derive the equations needed for the calculation of local optical absorption spectra.

\section{A. Single-particle states}

In semiconductor QDs, carriers are confined in all three space directions. To simplify our analysis, we assume that a suitable parameterization of the dot confinement potential is known (e.g., from experiment) and that the confinement potential varies sufficiently slow on the length scale of the lattice constant. We thus shall make use of the envelope-function approach [32]; moreover, since the energy region of our present concern is relatively close to the semiconductor band gap, we describe the material band structure in terms of a single electron and hole band within the usual effective-mass approximation. More specifically, the envelope-function equation for single electrons and holes reads:

$$
\left(-\frac{\hbar^{2} \nabla^{2}}{2 m_{e, h}}+V_{c}^{e, h}(\mathbf{r})\right) \phi_{\mu}^{e, h}(\mathbf{r})=\epsilon_{\mu}^{e, h} \phi_{\mu}^{e, h}(\mathbf{r})
$$

where $m_{e}\left(m_{h}\right)$ is the effective mass and $V_{c}^{e}\left(V_{c}^{h}\right)$ is the confinement potential energy for electrons (holes). Following our approach developed earlier [33], we numerically solve Eq. (2.1) for arbitrary confinement potentials by use of a plane-wave expansion with periodic boundary conditions (see Appendix A).

\section{B. Exciton states}

When the dot structure is perturbed by an external light field (e.g., laser), electron-hole pairs are created which propagate in the presence of the mutual Coulomb interaction and of the dot confinement potential. Within the present paper, we shall restrict ourselves to the linear optical response, i.e., the dynamics of a single electronhole pair. Then, the exciton dynamics is described by the electron-hole wavefunction $\Psi\left(\mathbf{r}_{e}, \mathbf{r}_{h}\right)$, with the squared modulus being the probability of finding the electron at position $\mathbf{r}_{e}$ when the hole is at position $\mathbf{r}_{h}$.

If we expand the electron-hole ("exciton") eigenfunction in terms of single-particle states viz.:

$$
\Psi^{\lambda}\left(\mathbf{r}_{e}, \mathbf{r}_{h}\right)=\sum_{\mu \nu} \phi_{\mu}^{e}\left(\mathbf{r}_{e}\right) \Psi_{\mu \nu}^{\lambda} \phi_{\nu}^{h}\left(\mathbf{r}_{h}\right)
$$

we obtain the excitonic eigenvalue problem [34,35]:

$$
\left(\epsilon_{\mu}^{e}+\epsilon_{\nu}^{h}\right) \Psi_{\mu \nu}^{\lambda}+\sum_{\mu^{\prime} \nu^{\prime}} V_{\mu \mu^{\prime}, \nu \nu^{\prime}}^{e h} \Psi_{\mu^{\prime} \nu^{\prime}}^{\lambda}=E_{\lambda} \Psi_{\mu \nu}^{\lambda}
$$

As will be shown in the following, the exciton spectrum $E_{\lambda}$ directly provides the optical transition energies whereas the excitonic wavefunctions $\Psi^{\lambda}$ determine the oscillator strengths of the corresponding transitions. In Eq. (2.3) we have introduced the electron-hole Coulomb matrix elements [36]:

$$
V_{\mu \mu^{\prime}, \nu \nu^{\prime}}^{e h}=-e^{2} \int d \mathbf{r}_{e} d \mathbf{r}_{h} \frac{\phi_{\mu}^{e^{*}}\left(\mathbf{r}_{e}\right) \phi_{\mu^{\prime}}^{e}\left(\mathbf{r}_{e}\right) \phi_{\nu}^{h^{*}}\left(\mathbf{r}_{h}\right) \phi_{\nu^{\prime}}^{h}\left(\mathbf{r}_{h}\right)}{\kappa_{o}\left|\mathbf{r}_{e}-\mathbf{r}_{h}\right|}
$$

where $e$ is the elementary charge and $\kappa_{O}$ is the static dielectric constant of the bulk semiconductor (note that in Eq. 2.4 we have not considered the electron-hole exchange interaction). Within our computational approach, we consider in Eq. 2.3 typically a basis of 12 states for electrons and holes, respectively, and obtain the excitonic eigenfunctions by direct diagonalization of the Hamiltonian matrix.

\section{Local optical absorption}

When the semiconductor nanostructure is excited by a local near-field probe, the total absorbed power $\alpha(\omega)$ at a given frequency $\omega$ is proportional to $\int d \mathbf{r} \mathcal{E}_{\omega}(\mathbf{r}) P(\mathbf{r}, \omega)$, where $\mathcal{E}_{\omega}(\mathbf{r})$ is the electro-magnetic field distribution of the near-field probe. Within linear response, the induced interband polarization $P(\mathbf{r}, \omega)$ is related to $\mathcal{E}_{\omega}(\mathbf{r})$ through:

$$
P(\mathbf{r}, \omega)=\int d \mathbf{r}^{\prime} \chi\left(\mathbf{r}, \mathbf{r}^{\prime} ; \omega\right) \mathcal{E}_{\omega}\left(\mathbf{r}^{\prime}\right)
$$


where the non-local electrical susceptibility $\chi\left(\mathbf{r}, \mathbf{r}^{\prime} ; \omega\right)$ can be expressed in terms of the excitonic eigenenergies and eigenfunctions [24]:

$$
\chi\left(\mathbf{r}, \mathbf{r}^{\prime} ; \omega\right)=\mu_{o}^{2} \sum_{\lambda} \frac{\Psi^{\lambda}(\mathbf{r}, \mathbf{r}) \Psi^{\lambda^{*}}\left(\mathbf{r}^{\prime}, \mathbf{r}^{\prime}\right)}{E_{\lambda}-\hbar \omega-i \gamma} .
$$

Here, $\mu_{o}$ is the dipole-matrix element of the bulk semiconductor, while we have introduced a small damping constant $\gamma$ accounting for the finite lifetime of exciton states due to environment coupling (e.g., phonons). To derive our final expression, it turns out to be convenient to consider for the elctromagnetic field distribution a given profile $\xi$ centered around the beam position $\mathbf{R}$, i.e., $\mathcal{E}_{\omega}(\mathbf{r})=\mathcal{E}_{\omega} \xi(\mathbf{r}-\mathbf{R})$. Then, the local spectrum for a given tip position $\mathbf{R}$ can be expressed in the form 24] (see also Appendix A):

$$
\alpha_{\xi}(\mathbf{R}, \omega) \propto \Im \sum_{\lambda} \frac{\alpha_{\xi}^{\lambda}(\mathbf{R})}{E_{\lambda}-i \gamma-\hbar \omega}
$$

where

$$
\alpha_{\xi}^{\lambda}(\mathbf{R})=\left|\int d \mathbf{r} \Psi^{\lambda}(\mathbf{r}, \mathbf{r}) \xi(\mathbf{r}-\mathbf{R})\right|^{2} .
$$

Two limiting cases can be identified. For a spatially homogeneous electromagnetic field (far-field), the oscillator strength $\alpha_{\xi}^{\lambda}$ is given by the spatial average of the excitonic wavefunction, i.e., $\alpha_{\xi}^{\lambda}(\mathbf{R})=\left|\int d \mathbf{r} \Psi^{\lambda}(\mathbf{r}, \mathbf{r})\right|^{2}$. In the opposite (and hypothetical) limit of an infinitely narrow probe, $\xi(\mathbf{r}-\mathbf{R})=\delta(\mathbf{r}-\mathbf{R})$, one is probing the local value of the exciton wavefunction, i.e., $\alpha_{\xi}^{\lambda}(\mathbf{R})=$ $\left|\Psi^{\lambda}(\mathbf{R}, \mathbf{R})\right|^{2}$. Finally, within the intermediate regime of a narrow but finite probe, $\Psi^{\lambda}(\mathbf{r}, \mathbf{r})$ is averaged over a region which is determined by the spatial extension of the light beam; therefore, excitonic transitions which are optically forbidden in the far-field may become visible in the near-field.

\section{RESULTS}

In the following sections we consider the interaction of the EM field with excitonic states of single and double QDs; for the latter system, we focus particularly on the transition between two isolated QDs and a the "artificial molecule", where the electronic states of two QDs are strongly overlapping.

\section{A. Single-particle states}

We shall consider a prototypical QD confinement which is composed of a $2 \mathrm{D}$ harmonic potential in the $(x, y)$-plane and a rectangular quantum well along $z ;$ such confinement potentials have been demonstrated to be a good approximation for self-assembled quantum dots formed by strained-layer epitaxy. We focus on cases where the $z$-confinement is stronger than the $(x, y)$ one, so that the confinement potential can be written as

$$
V_{c}^{e, h}(x, y, z)=V_{\|}^{e, h}(x, y)+V_{o}^{e, h} \theta\left(|z|-\frac{z_{o}}{2}\right),
$$

where $z_{o}$ is the width of the quantum-well and $V_{o}^{e, h}$ the band offsets for electrons and holes, respectively. For a single dot the in-plane confinement potential $V_{\|}^{e, h}(x, y)$ is of the form:

$$
V_{\|}^{e, h}(x, y)=\frac{1}{2} \mathcal{K}_{e, h}\left(x^{2}+y^{2}\right),
$$

while for two dots (i.e., double dot) separated by the distance $d$ :

$$
V_{\|}^{e, h}(x, y)= \begin{cases}\frac{1}{2} \mathcal{K}_{e, h}\left(\left(|x|-\frac{d}{2}\right)^{2}+y^{2}\right) & \text { for }|x|>\frac{d}{4} \\ \frac{1}{2} \mathcal{K}_{e, h}\left(\left(\frac{d^{2}}{8}-x^{2}\right)+y^{2}\right) & \text { otherwise }\end{cases}
$$

with $\mathcal{K}_{e, h}=m_{e, h}\left(\omega_{o}^{e, h}\right)^{2}$, and $\hbar \omega_{o}^{e, h}$ the level splittings of the in-plane harmonic potential. The shape of the double-dot potential has been obtained by matching the parabolas with opposite curvature, such that the potential is continuous and smooth at $x= \pm \frac{d}{4}$; the shape of the resulting potential along the $x$ - direction is shown, for selected inter-dot distances $d$, in Figs. 1(b), 1(d). Material and dot parameters which are used in this paper are listed in Table I; with this choice of parameters, electron and hole wavefunctions have approximately the same lateral extension, and the QW-induced intersubband splittings are much larger than $\hbar \omega_{o}^{e}$ and $\hbar \omega_{o}^{h}$.

With our choice of the confinement potential, Eq. (refeq:total.confinement), the single-particle energies of a $\mathrm{QD}$ are $E_{\mathrm{QD}}=E_{\mathrm{QW}}+E_{\mathrm{harm}}$, where $E_{\mathrm{QW}}$ is the confinement energy of the QW along $z$ and $E_{\text {harm }}$ is the confienment energy of the 2D single- or double-harmonic potential. Single-particle energies and envelope functions have been computed numerically within a plane-wave scheme. However, for a single QD the 2D eigenstates can be found analytically and are the well-known "FockDarwin" states [1] (we stress, however, that the extension in the $z$-direction is of crucial importance for the calculation of the Coulomb matrix elements and the optical properties, and unavoidably has to be taken into account in any realistic calculation; see also discussion in Ref. [37]). For such states $E_{\text {harm }}=(n+1) \hbar \omega_{o}^{e, h}$, where $n=0,1, \ldots$ is the principal quantum number, and each level is $(n+1)$-fold degenerate; in Table I w we summarize for convenience some properties of these "Fock-Darwin" states [1]. 
Figure 1 shows the calculated single-particle energies for electrons and holes for the more complex case of a double QD with the confinement potential given in Eq. (3.3) and with parameters listed in Table The The lower panels (Figs. 1(b,d)) show the confinement potentials for electrons and holes at selected inter-dot distances. Obviously, for large dot separations $d$ ( $d \gtrsim 60 \mathrm{~nm}$ ) the system can be well approximated by two separate QDs; in this regime the equidistance of the excited states and the correct degeneracy of the Fock-Darwin states is obtained. When $d$ is small enough that carriers have sufficient energy to overcome (or tunnel through) the barrier between the two dots, the degeneracy is removed, and the energy levels have a non-monotonous behaviour which reflects the transition from two separated carrier systems to a single one, and is similar to the one found, e.g. for coupled QWs [38]. For the smallest dot distances the doubledot potential merges into a single-dot potential, and the Fock-Darwin states of a single dot are recovered.

\section{B. Role of the Coulomb correlation in the far-field spectra}

Before turning to the analysis of near-field spectra, we shortly discuss the limiting case of very broad EM field distribution (far-field spectra). This discussion allows us to elucidate the role of the electron-hole Coulomb correlation, particularly in the transition from two separate "artificial atoms" to an "artificial molecule".

Far-field spectra can be obtained in the formalism of Sec. II C with a spatially homogeneous electromagnetic field distribution probe $\xi$ ):

$$
\alpha_{\xi(\mathbf{r})=\mathrm{const}}^{\lambda}=\left|\int d \mathbf{r} \Psi^{\lambda}(\mathbf{r}, \mathbf{r})\right|^{2} .
$$

Figs. 2 and 3 show the calculated far-field spectra for a double QD as a function of the dot distance $d$. We first concentrate on the calculations where Coulomb correlations were artificially set to zero (Figs. 2(a) and 3(a)): Because of symmetry, only a small fraction of all possible electron-hole transitions is visible; from Eq. (3.4) and using $\int d \varphi \exp i\left(m_{e}+m_{h}\right) \varphi \propto \delta_{m_{e},-m_{h}}$ we obtain that optical transitions are only allowed between electron and hole single-particle states with opposite angular momentum. Indeed, for large distances (uncoupled QDs) only three strong absorption peaks are observed, with an energy splitting of approximately $\hbar \omega_{o}^{e}+\hbar \omega_{o}^{h}$; the intensity of the peaks increases with energy (with ratio $1: 2: 3$ ). These can be attributed to transitions between electrons and holes single-particle states (see Table II) of the $1 \mathrm{~s}$ symmetry (peak at $\approx 70 \mathrm{meV}$ ), the $1 p$ symmetry (peak at $\approx 95 \mathrm{meV}$ ), and the $1 d$ and $2 s$ symmetries (peak at $\approx 120 \mathrm{meV})$.

When symmetry is reduced, either because of an asymmetric confinement potential or by the presence of an external inhomogeneous EM-field (as will be discussed later), the selection rules noted above are relaxed. Indeed, when $d$ is reduced and the two QDs begin to interact, the calculated spectra show a much richer structure, as shown in Fig. 2(a) and 3(a), reflecting the reduction of built-in symmetry. Obviously, when $d \simeq 0$, the usual selection rules of a single-QD are recovered.

When Coulomb interaction is included, inspection of the exciton wavefunctions $\Psi_{\mu \nu}^{\lambda}$ (obtained from the solutions of Eq. (2.3)) shows that a number of different single-particle transitions contributes to each excitonic state [39]. Coulomb interaction affects the optical spectra [see Figs. 3(a) and 3(b)] in several ways. Firstly, because of the attractive electron-hole interaction leading to the "bound" excitonic states, we observe a red-shift of the peaks; peak separation is barely affected, however, at least for large $d$. Secondly, we observe a redistribution of the oscillator strength; for isolated QDs the three main peaks are of similar height. In general, oscillator strength is transferred from higher to lower peaks. This effect is particularly strong, e.g., in the doublet which splits from the lowest peak when the two QDs approach; contrary to the uncorrelated case, the heighest partner is very weak. Finally, Coulomb interaction is responsible for the appearance of additional lines (see, e.g., for $d=70$ $\mathrm{nm}$ the peak at $70 \mathrm{meV}$ ). While the first two effects (redshift and transfer of oscillator strength) are similar to what is found in the absorption spectra of semiconductor quantum wires, and thus can be considered as a general fingerprint of Coulomb correlations in the optical properties of semiconductor nanostructures, the origin of the additional peaks is best discussed in connection with the calculated near-field optical spectra and, therefore, is postponed to the next section.

\section{Optical near-field spectra}

In this section we discuss the local absorption spectra of single and coupled QDs. Because of the narrow well width of the dot confinement potential (see Table I) , the EM profile of the near-field probe along $z$ has an only minor influence on the results, and we use:

$$
\xi(x, y, z) \propto \exp \left(-\frac{x^{2}+y^{2}}{2 \sigma^{2}}\right) .
$$

The spatial resolution of the electromagnetic field distribution of Eq. (3.5) is then approximately given by the full-width at half maximum (FWHM) of the Gaussian (i.e., $2 \sqrt{2 \ln 2} \sigma \approx 2.35 \sigma$ ). Since the Gaussian acts as an envelope on $\Psi^{\lambda}$, in the intermediate regime of a narrow but finite $\sigma$ the spatial average only extends over the region where the Gaussian is non-vanishing.

Since the extension of the quantum states under investigation is of the order of a few tens of nano-meters (see also Figs. 5 and 8, to be discussed below), in our 
calculations we consider three different regimes of spatial resolution: $i$ ) a regime where the FWHM is much larger than the extension of the quantum states (as a characteristic value we use $\sigma=50 \mathrm{~nm}$ ); ii) a regime where the FWHM is comparable to the extension of the relevant quantum states (we use $\sigma=10 \mathrm{~nm}$ ); iii) a regime with an extremely narrow probe beam (we use $\sigma=0.1 \mathrm{~nm}$ ). Calculations performed in this latter (unphysical) regime are used for illustrative purposes to obtain a "cartography" of the exciton wavefunction, as discussed at the end of Sec. II G. We finally notice that the excitonic Bohr radius $\approx 12 \mathrm{~nm}$ for GaAs.

\section{Single quantum dot}

In Fig. 4 we report the calculated local absorption spectra $\alpha_{\xi}(X, \hbar \omega)$ for a single QD as a function of the tip position. The tip is swept along one direction, passing through the center of the QD.

In Figs. 4(a-c) we show the calculated spectra neglecting Coulomb interaction. For the highest spatial resolution [Fig. 4(a)], the local absorption at photon energy $E_{\lambda}$ is proportional to $\left.\int d z \Psi^{\lambda}(\mathbf{r}, \mathbf{r})\right|_{y=0}$. Given the energy splitting $\hbar \omega_{o}^{h}=3.5 \mathrm{meV}$ for holes and $\hbar \omega_{o}^{e}=20$ meV for electrons, we can attribute the triplet of peaks at $\approx 70 \mathrm{meV}$ to the single-particle transitions involving the $1 s$ state of electrons and the $1 s, 1 p$, and $(2 s, 1 d)$ states (in order of increasing energy) of holes (see also Table II); analogously, the triplet at $\approx 90 \mathrm{meV}$ is attributed to the transitions involving the $1 p$ state of electrons and the $1 s, 1 p$, and $(2 s, 1 d)$ hole states; indeed, in Fig. 4(a) the localization of the absorption peaks is suggestive of the $s$-, $p$ - or $d$-type symmetry of the corresponding FockDarwin states. These features are still present at the intermediate resolution [Fig. 4(b)], but disappear at the opposite limit of a broad probe [Figs. 4(c)]. This is expected, since, when a localized EM-field is present, the symmetry of the whole system (nanostructure+EM-field) is lower than that of the nanostructure (except when the probe is centered in the symmetry center of the structure), and far-field selection rules are relaxed. When the probe is broadened, however, the built-in symmetry of the structure is recovered, and optical far-field selection rules (i.e., optical transitions only between electron and hole states with opposite angular momentum $m$ ) apply; therefore, the spectra are almost identical to those of two separated dots in far-field spectroscopy, already discussed in Figs. 2 and 3.

When we compare Figs. 4(a-c) with Figs. 4(d-f), we find that Coulomb interaction induces several effects which are expected on the basis of the discussion of the far-field spectra. In particular, we find i) an almost rigid redshift of the spectra; ii) a transfer of oscillator strength from transitions at higher energies to those at lower energies; iii) the appearance of new features in the optical spectra. To discuss the origin of these new opti- cal features caused by Coulomb interactions, let us consider, e.g., the optical peaks at photon energy $\approx 65 \mathrm{meV}$ (Figs. $4(\mathrm{~d}-\mathrm{f}))$ : They are quite strong at $\sigma=0.1 \mathrm{~nm}$ (Fig. 4(d)), almost disappear at $\sigma=10 \mathrm{~nm}$ (Fig. 4(e)), and are visible again in the far field limit (Fig. 4(f)). Such a behaviour is rather unexpected and noticeably differs from that of other transitions, which - with increasing $\sigma$ - either remain strong or gradually disappear due to symmetry reasons, as discussed above. To investigate the origin of this non-monotonic dependence, in the following we analyze the three excitons within the corresponding energy range. Figure 5 shows a contour plot of the respective exciton wavefunction $\left.\Psi^{\lambda}(\mathbf{r}, \mathbf{r})\right|_{z=0}$. Apparently, in Fig. 5(a) the exciton has $s$-type symmetry, whereas the other two electron-hole states have $p$ type symmetry. (Because of the periodicity box used in our calculations, the two-fold degenerate $p$-type exciton wavefunctions have cartesian rather than cylinder symmetry; note that, since the presence of the near-field tip destroys the cylinder symmetry, the wavefunctions shown in Fig. 5 indeed form a natural basis; see also Table III). Next, we note that the average $\int d \mathbf{r} \Psi^{\lambda}(\mathbf{r}, \mathbf{r})$ of the $p$-type exciton wavefunctions is zero. Since with increasing $\sigma$ the radius within which the exciton eigenfunctions $\Psi^{\lambda}$ are averaged increases, we expect for these $p$-type functions with increasing $\sigma$ a monotonically decreasing behavior. The exciton shown in Fig. 5(a), on the other hand, has a non-zero average and is therefore visible in both the optical far- and near-field. A closer inspection of the exciton wavefunction $\Psi_{\mu \nu}^{\lambda}$ reveals that the largest contribution stems from the transition between the $1 s$ state of electrons and the $2 s$ state of holes, but there is also a noticeable contribution from the $1 s$ $1 s$ and $1 p-1 p$ electron-hole transitions. Indeed, only the latter contributions couple in the far-field to the light field. In the regime of finite resolution, there is an optimal cancellation when the FWHM of the EM near field (i.e. $2 \sqrt{2 \ln 2} \sigma \approx 2.35 \sigma$ ) becomes equal to the Bohr radius. This is clearly depicted in Fig. 6, where, in order to facilitate our discussion, we have introduced the quantity $I_{\xi}^{\lambda} \propto \int d \mathbf{R} \alpha_{\xi}^{\lambda}(\mathbf{R})$, which provides a measure of the relative contribution of each exciton to the absorption spectra. Fig. 6 shows $I_{\xi}^{\lambda}$ for the three excitons (shown Fig. 5) within the energy region of $65 \mathrm{meV}$ : We observe that with increasing $\sigma$, the $p$-type functions (open circles) indeed vanish monotonically, whereas for the $s$-type exciton (full circles) there exists an optimal cancellation when the FWHM of the EM field distribution becomes approximately equal to the Bohr radius. In spite of the specific carrier states of a single parabolic QD, we expect that such non-monotonic behavior appears quite generally in semiconductor nanostructures where carrier states are confined on a length scale comparable to the Bohr radius, and thus provides a striking fingerprint of Coulomb correlations in the optical near-field spectra (we find similar behavior in our calculations for the near-field spectra of coupled QDs discussed below). 


\section{Double quantum dot}

In Figure 7 we show the calculated local absorption spectra $\alpha_{\xi}(X, \hbar \omega)$ for a double QD for selected values of the interdot distance and $\sigma$. The tip of the probe is swept along the direction which passes through the centers of the two QDs.

Let us first concentrate on the results with $\sigma=0.1$ $\mathrm{nm}$ and with the Coulomb interaction taken into account (Figs. $7(\mathrm{~d}, \mathrm{~g}, \mathrm{j}))$. With decreasing interdot distance we observe the transition from a system where the energetically lowest exciton states are almost localized in the spatially separated minima of the two dots, to a system where the electron-hole states extend over the whole nanostructure. Here, the $s$-like ground-state excitons of Fig. $7(\mathrm{j})$ split up into a "bonding" and an "anti-bonding" state (Fig. 7(d)). By comparing Figs. 7(d) and 7(f), we find that in the optical far-field only the symmetric ground state exciton couples to the light field.

Next, we discuss the optical features at the photon energy of $\approx 70 \mathrm{meV}$ for $d=40 \mathrm{~nm}$. As in the case of the single dot, these features show a non-monotonic dependence on the probe width. As can be inferred from the calculations with $\sigma=0.1 \mathrm{~nm}$, there are several excitonic states contributing to the spectral features in this energy range; Fig. 8 shows the excitonic wavefunction of two states out of the six states with $E_{\lambda} \approx 70 \mathrm{meV}$ for for $d=40 \mathrm{~nm}$; it can be inferred that for a spatial resolution of the near-field probe comparable to the excitonic Bohr radius $(\approx 12 \mathrm{~nm})$ there is again an optimal cancellation. This is a remarkable finding, because it clearly demonstrates that such a behaviour indeed is a general characteristics of semiconductor nanostructures, and does not depend on peculiar symmetries of the confining potential.

\section{SUMMARY AND CONCLUSIONS}

We have analyzed theoretically the interaction between a model near-field probe and a zero-dimensional heterostructure: Quantum confinement of the electron and hole states, as well as their Coulomb interaction in the linear regime are fully included in our description.

We have specifically considered single and coupled semiconductor quantum dots, and shown that absorption is strongly influenced by the spatial interference of the exciton wavefunctions, which depends on the spatial extension of the light beam. As a consequence, near-field experiments on quantum dots are predicted to display unexpected spectral features whose dependence on spatial resolution is highly non-trivial.

When combined with an appropriate choice of the EM field distribution, our approach provides the necessary tool for interpretation of near-field absorption spectra of quantum dots as the spatial resolution of experiments becomes comparable with the Bohr radius of the exciton in the nanostructure.

\section{ACKNOWLEDGMENTS}

We thank Fausto Rossi for most stimulating discussions. This work was supported in part by INFM through PRA-99-SSQI, and by the EC under the TMR Network "Ultrafast Quantum Optoelectronics" and the IST programme "SQID". U.H. acknowledges support by the EC through a TMR Marie Curie Grant.

\section{APPENDIX A: PLANE-WAVE APPROACH}

In this Appendix we discuss details of our numerical solution schemes based on a plane-wave expansion. Following our approach developed earlier [33], we consider the problem of a single or double QD which is located inside a box with periodic boundary conditions, where the boxsize is chosen sufficiently large to avoid interactions with "neighbour" dots. As a complete set of functions, inside the periodicity box we use a plane-wave basis, $|\mathbf{k}\rangle$, with:

$$
k_{\alpha}=\frac{2 \pi n_{\alpha}}{L_{\alpha}}, \quad n_{\alpha} \in \mathcal{Z}, \quad \alpha=x, y, z
$$

Here $L_{\alpha}$ denotes the sizes of the periodicity box (we use the same box for electrons and holes). We next expand the single-particle wavefunctions for electrons and holes within the plane-wave basis:

$$
\tilde{\phi}_{\mu, \mathbf{k}}^{e, h}=\Omega^{-1} \int d \mathbf{r} e^{-i \mathbf{k} \cdot \mathbf{r}} \phi_{\mu}^{e, h}(\mathbf{r})
$$

with $\Omega$ the volume of the periodicity box. The envelopefunction equation (2.1) is then transformed to:

$$
\sum_{\mathbf{k}^{\prime}}\left(\frac{\hbar^{2} \mathbf{k}^{2}}{2 m_{e, h}} \delta_{\mathbf{k k}^{\prime}}+\tilde{V}_{c, \mathbf{k}-\mathbf{k}^{\prime}}^{e, h}\right) \tilde{\phi}_{\mu, \mathbf{k}^{\prime}}^{e, h}=\epsilon_{\mu}^{e, h} \tilde{\phi}_{\mu, \mathbf{k}}^{e, h}
$$

which can be solved by standard diagonalization techniques. To keep the numerics tractable, only wavevectors smaller than a given cut-off wavevector are considered (typically 2000-3000 wavevectors). In our computational approach, we perform the Fourier transform of the confinement potential by storing $V_{c}^{e, h}(\mathbf{r})$ on an appropriate grid (with a typical number of 30 points along each direction), and approximating within each cube $V_{c}^{e, h}(\mathbf{r})$ by its average value.

In the calculation of the near-field spectra, we define the electron-hole index $l=(\mu, \nu)$. Then:

$$
\Psi^{\lambda}(\mathbf{r}, \mathbf{r})=\sum_{l} \Psi_{l}^{\lambda} \phi_{\mu_{l}}^{e}(\mathbf{r}) \phi_{\nu_{l}}^{h}(\mathbf{r})
$$


and we obtain for $\alpha_{\xi}^{\lambda}(\mathbf{R})$ of Eq. (2.8) the final result:

$$
\alpha_{\xi}^{\lambda}(\mathbf{R})=\left|\sum_{l} \Psi_{l}^{\lambda} \sum_{\mathbf{k}, \mathbf{k}^{\prime}} \tilde{\xi}_{\mathbf{k}+\mathbf{k}^{\prime}}(\mathbf{R}) \tilde{\phi}_{\mu_{l}, \mathbf{k}}^{e} \tilde{\phi}_{\nu_{l}, \mathbf{k}^{\prime}}^{h}\right|^{2}
$$

with $\tilde{\xi}_{\mathbf{k}}(\mathbf{R})=\Omega^{-1} \int d \mathbf{r} \xi(\mathbf{r}) e^{i \mathbf{k} \cdot(\mathbf{r}+\mathbf{R})}$.

[1] L. Jacak, P. Hawrylak, and A. Wojs, Quantum Dots (Springer, Berlin, 1998).

[2] D. Bimberg, M. Grundmann, and N. Ledentsov, Quantum Dot Heterostructures (Wiley, New York, 1998).

[3] A. Zrenner, L. V. Butov, M. Hagn, G. Abstreiter, G. Böhm, and G. Weimann, Phys. Rev. Lett. 72, 3382 (1994) ; K. Brunner, G. Abstreiter, G. Böhm, G. Tränkle, G. Weimann, ibid. 73, 1138 (1994).

[4] D. Gammon, E. S. Snow, B. V. Shanabrook, D. S. Kratzer, D. Park, Phys. Rev. Lett. 76, 3005 (1996).

[5] H. F. Hess, E. Betzig, T. D. Harris, L. N. Pfeiffer, K. W. West, Science 264, 1740 (1994).

[6] F. Flack, N. Samarth, V. Nikitin, P. A. Crowell, J. Shi, J. Levy, and D. D. Awschalom, Phys. Rev. B 54, R17312 (1996).

[7] J.-Y. Marzin, J.-M. Gérard, A. Izraël, D. Barrier, G. Bastard, Phys. Rev. Lett. 73, 716 (1994).

[8] M. Grundmann, J. Christen, N.N. Ledentsov, J. Böhrer, D. Bimberg, S.S. Ruvimov, P. Werner, U. Richter, U. Gösele, J. Heydenreich, V.M. Ustinov, A.Y. Egurov, A.E. Zhurov, P.S. Kop'ev, and Z.I. Alferov, Phys. Rev. Lett. 74, 4043 (1995).

[9] R. Leon, P. M. Petroff, D. Leonard, S. Fafard, Science 267, 1966 (1995).

[10] A. Hartmann, Y. Ducommun, E. Kapon, U. Hohenester, and E. Molinari, Phys. Rev. Lett. (2000), in press.

[11] C. Obermüller, A. Deisenrieder, G. Abstreiter, G. Karrai, S. Grosse, S. Manus, J. Feldmann, H. Lipsanen, M. Sopanen, and J. Ahopelto, Appl. Phys. Lett. 74, 3200 (1999); R. Rinaldi, S. Antonaci, M. DeVittorio, R. Cingolani, U. Hohenester, E. Molinari, H. Lipsanen, J. Tulkki, Phys. Rev. B (2000), in press.

[12] W. Wegscheider, G. Schedelbeck, G. Abstreiter, M. Rother, M. Bichler, Phys. Rev. Lett. 79, 1917 (1997); G. Schedelbeck, W. Wegscheider, M. Bichler, G. Abstreiter, Science 278, 1792 (1997).

[13] C. B. Murray, C. R. Kagan, M. G. Bawendi, Science 270, 1335 (1995).

[14] A. P. Alivisatos, Science 271, 933 (1996).

[15] M.A. Paesler and P.J. Moyer, Near-Field Optics: Theory, Instrumentation, and Applications (Wiley, New York, 1996).

[16] T. Saiki and K. Matsuda, Appl. Phys. Lett. 74, 2773 (1999).

[17] E. Betzig, J. K. Trautman, T. D. Harris, J. S. Weiner, and R. L. Kostelak, Science 251, 1468 (1991)
[18] T. D. Harris, D. Gershoni, R. D. Grober, L. Pfeiffer, K. West, and N. Chand, Appl. Phys. Lett. 68, 988 (1996).

[19] A. Richter, G. Behme, M. Suptitz, Ch. Lienau, T. Elsaesser, M. Ramsteiner, R. Notzel, and K. Ploog, Phys. Rev. Lett. 79, 2145 (1997).

[20] L. Landin, M. S. Miller, M.-E. Pistol, C. E. Pryor, and L. Samuelson, Science 280, 262 (1998).

[21] A. Chavez-Pirson, J. Temmyo, H. Kamada, H. Gotoh, and H. Ando, Appl. Phys. Lett. 72, 3494 (1998).

[22] Y. Toda, M. Kourogi, M. Ohtsu, Y. Nagamune, and Y. Arakawa, Appl. Phys. Lett. 69, 827 (1996); Y. Toda, O. Moriwaki, M. Nishioka, and Y. Arakawa, Phys. Rev. Lett. 82, 4114 (1999).

[23] T. Matsumoto, M. Ohtsu, K. Matsuda, T. Saiki, H. Saito, and K. Nishi, Appl. Phys. Lett. 75, 3246 (1999).

[24] O. Mauritz, G. Goldoni, F. Rossi, and E. Molinari, Phys. Rev. Lett. 82, 847 (1999).

[25] M. Rontani, F. Rossi, F. Manghi, E. Molinari, Solid State Communications 112, 151 (1999), and references therein.

[26] See e.g. P. Zanardi and F. Rossi, Phys. Rev. Lett. 81, 4752 (1998); L. Quiroga and N.F. Johnson, Phys. Rev. Lett. 83, 2270 (1999); F. Troiani, U. Hohenester, and E. Molinari, submitted to Phys. Rev. B.

[27] R. Chang et al, J. Appl. Phys. 81, 3369 (1997).

[28] B. Hanewinkel, A. Knorr, P. Thomas, and S.W. Koch, Phys. Rev. B 55, 13715 (1997); G. von Freymann, Th. Schimmel, M. Wegener, B. Hanewinkel, A. Knorr, and S.W. Koch, Appl. Phys. Lett. 73, 1170 (1999).

[29] C. Girard, A. Dereux, and J.-C. Weber, Phys. Rev. E 58, 1081 (1998).

[30] G.W. Bryant, Appl. Phys. Lett. 72, 768 (1998); Ansheng Liu and G. Bryant, Phys. Rev. B 59, 2245 (1999).

[31] A. Chavez-Pirson and Sai Tak Chu, Appl. Phys. Lett. 74, 1507 (1999).

[32] P.Y. Yu and M. Cardona, Fundamentals of Semiconductors (Springer, Berlin, 1996).

[33] F. Rossi and E. Molinari, Phys. Rev. Lett. 76, 3642 (1996); Phys. Rev. B 53, 16462 (1996).

[34] H. Haug, S.W. Koch, Quantum Theory of the Optical and Electronic Properties of Semiconductors (World Scientific, Singapore, 1993).

[35] F. Rossi, Semicond. Sci. Technol. 13, 147 (1998).

[36] In our computational approach, we expand Eq. (2.4) in a plane-wave basis (see also Appendix A). Within this scheme, the $q^{-2}$-divergence due to the infinite range of the Coulomb potential has to be treated with some care. We assume that because of our use of the envelopefunction approximation the single-particle states $\phi^{e, h}$ for electrons and holes have an only small wavevector dependence (this assumption, in particular, holds for the low-energetic bound QD states of our present concern). We then replace the summation over $\mathbf{q}$ by an appropriate three-fold integration (i.e., we let the sizes of the periodicity box approach infinity) and numerically perform the remaining integration over the Fourier-transformed Coulomb potential; within spherical coordinates the $q^{-2}$ divergence is then precisely cancelled by the Jacobian of $d^{3} q$.

[37] M. Rontani, F. Rossi, F. Manghi, E. Molinari, Phys. 
Rev. B 59, 10165 (1999).

[38] C. D. Simserides and G. P. Triberis, J. Phys.: Condens. Matter 5, 6437 (1993).

[39] In our calculations, we use a basis of 12 electron and hole states, respectively (corresponding to a total number of 144 exciton states).

TABLE I. Material parameters for GaAs/AlGaAs and dot parameters which were used in the calculations. Here, $m_{o}$ is the free-electron mass.

\begin{tabular}{lll} 
description & value & units \\
\hline electron mass $m_{e}$ & 0.067 & $m_{o}$ \\
hole mass $m_{h}$ & 0.38 & $m_{o}$ \\
dielectric constant $\kappa_{o}$ & 12.9 & \\
conduction-band offset for electrons $V_{o}^{e}$ & 300 & $\mathrm{meV}$ \\
valence-band offset for holes $V_{o}^{h}$ & 200 & $\mathrm{meV}$ \\
confinement energy $\hbar \omega_{o}^{e}$ for electrons & 20 & $\mathrm{meV}$ \\
confinement energy $\hbar \omega_{o}^{h}$ for holes & 3.5 & $\mathrm{meV}$ \\
quantum-well width $z_{o}$ & 10 & $\mathrm{~nm}$ \\
\hline \hline
\end{tabular}

TABLE II. Eigenfunctions (Fock-Darwin states) with lowest energies for a particle with mass $\mu$ and for a potential of the form $V(x, y)=\frac{1}{2} \mu \omega_{o}^{2}\left(x^{2}+y^{2}\right)=\frac{1}{2} \mu \omega_{o}^{2} r^{2}$ (i.e., two-dimensional harmonic oscillator). We use $\mathcal{X}=x / a_{o}, \mathcal{Y}=y / a_{o}$, and $\mathcal{R}=r / a_{o}$, with $\left.a_{o}=\sqrt{(} \frac{\hbar}{\mu \omega_{o}}\right)$. Because of cylindrical symmetry, the angular momentum in the $z$-direction is a good quantum number $(m)$ and the angular part of the wavefunctions is of the form $\propto \exp \pm i m \varphi$; we use the notation $s$ for $m=0, p$ for $m= \pm 1$, and $d$ for $m= \pm 2$.

\begin{tabular}{|c|c|c|c|}
\hline Energy $\left(\hbar \omega_{o}\right)$ & $\begin{array}{r}\text { cartesian coordinates: } \\
\phi(\mathcal{X}, \mathcal{Y}) \propto \exp -\frac{1}{2}\left(\mathcal{X}^{2}+\mathcal{Y}^{2}\right) \\
\end{array}$ & $\begin{array}{r}\text { cylinder } \\
\phi(\mathcal{R}, \varphi) \propto \exp -\frac{1}{2} \mathcal{R}^{2} \\
\end{array}$ & notation \\
\hline 1 & $\times 1$ & $\times 1$ & $1 s$ \\
\hline 2 & $\begin{array}{l}\times \mathcal{X} \\
\times \mathcal{Y}\end{array}$ & $\times \mathcal{R} \exp \pm i \varphi$ & $1 p$ \\
\hline 3 & $\begin{array}{c}\times \mathcal{X Y} \\
\times\left(2 \mathcal{X}^{2}-1\right) \\
\times\left(2 \mathcal{Y}^{2}-1\right)\end{array}$ & $\begin{aligned} & \times\left(\mathcal{R}^{2}-1\right) \\
\times & \mathcal{R}^{2} \exp \pm 2 i \varphi\end{aligned}$ & $\begin{array}{l}2 s \\
1 d\end{array}$ \\
\hline
\end{tabular}



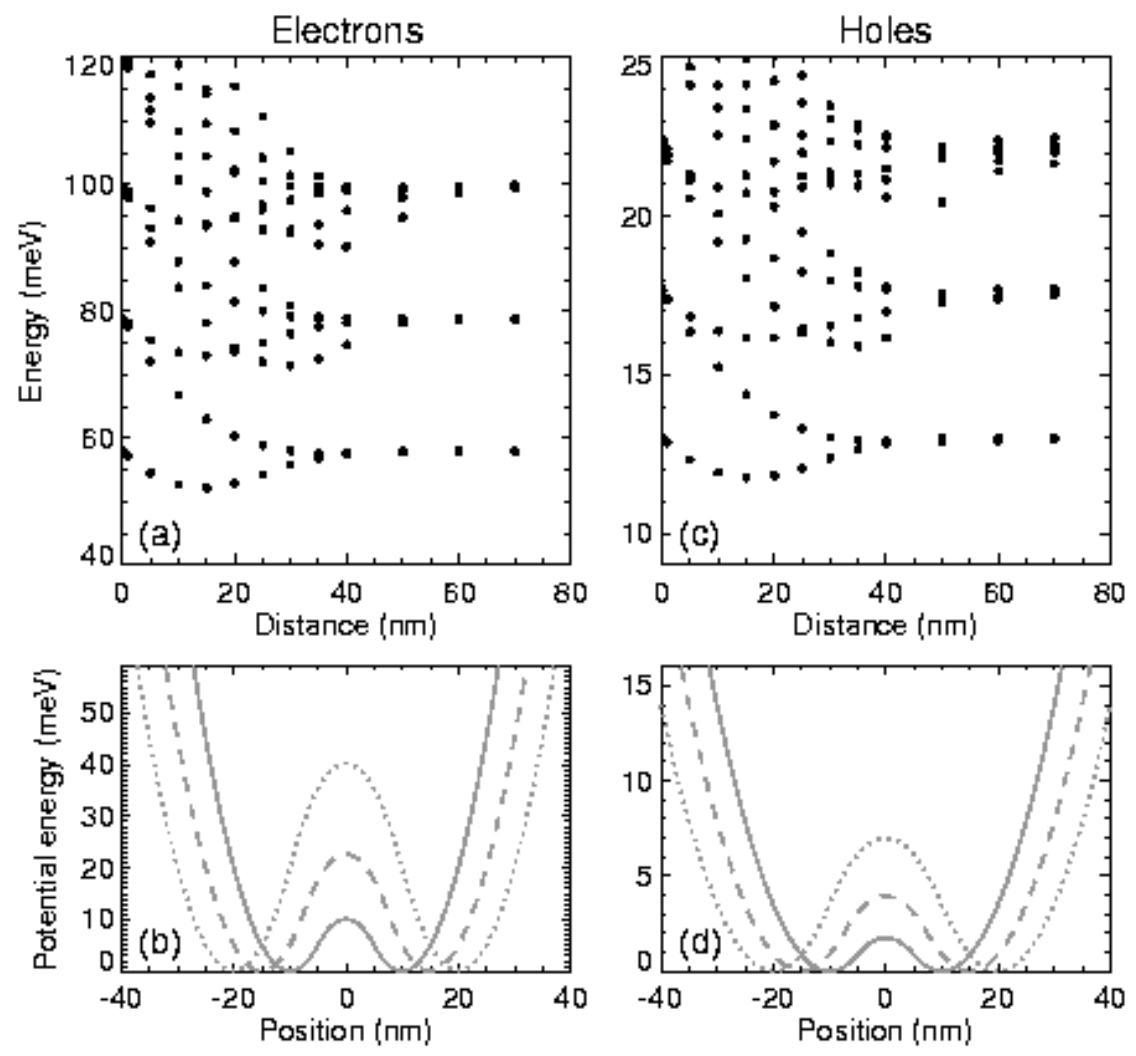

FIG. 1. Single-particle confinement energies as a function of the distance between the two dots, $d$ (upper panels) and the form of the confining potential along x-axis (lower panels) for $d=20 \mathrm{~nm}$ (solid line), $d=30 \mathrm{~nm}$ (dashed line) and $d=40 \mathrm{~nm}$ (dotted line). Left and right panels correspond to electrons and holes, respectively. 

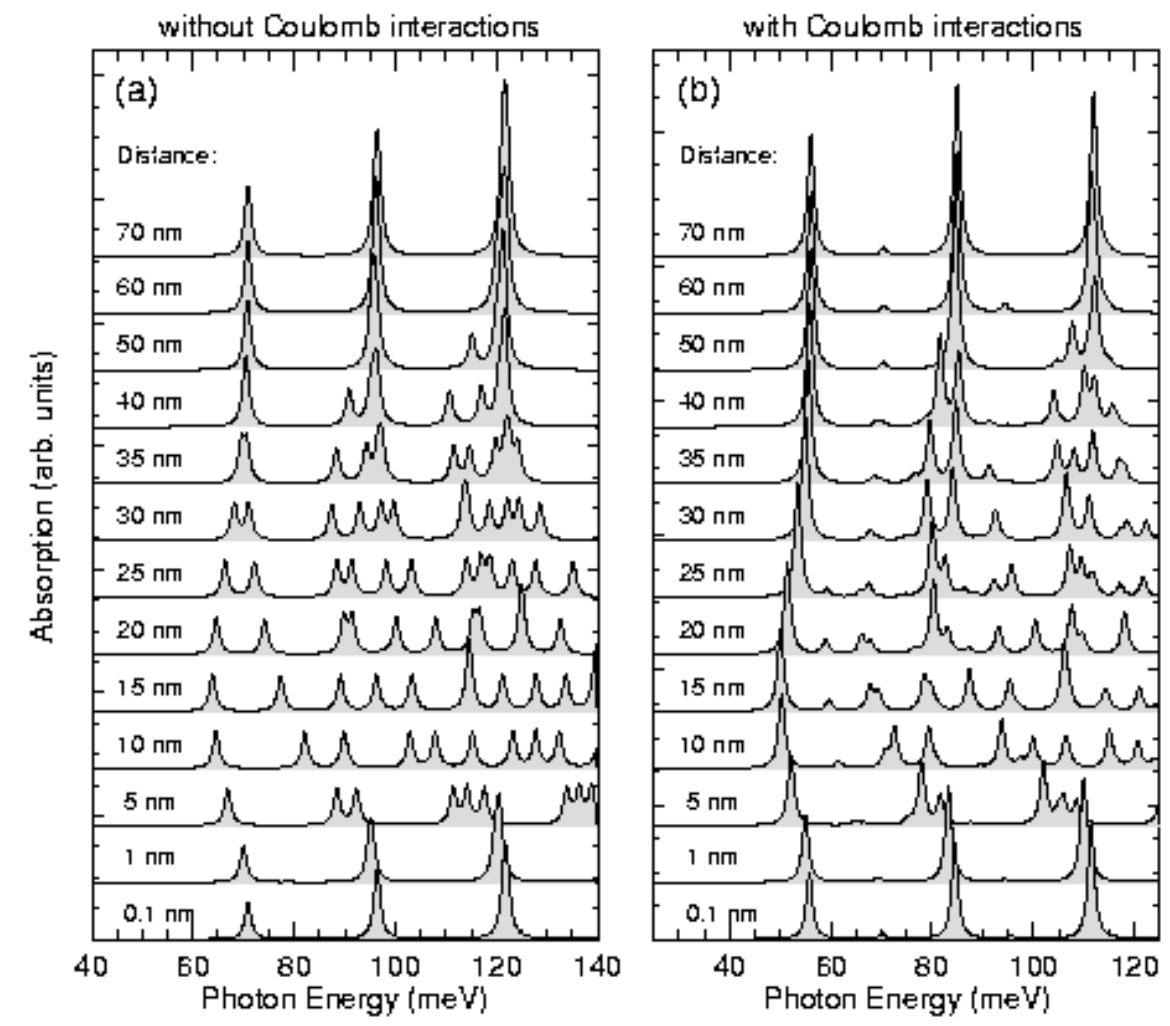

FIG. 2. Optical absorption spectra for a homogeneous electromagnetic field profile (i.e., far field) for a double quantum dot and for different distances $d$ : (a) Coulomb interactions neglected; (b) Coulomb interactions included. We use $\gamma=1$ meV. The photon energy is measured with respect to the bandgap. 

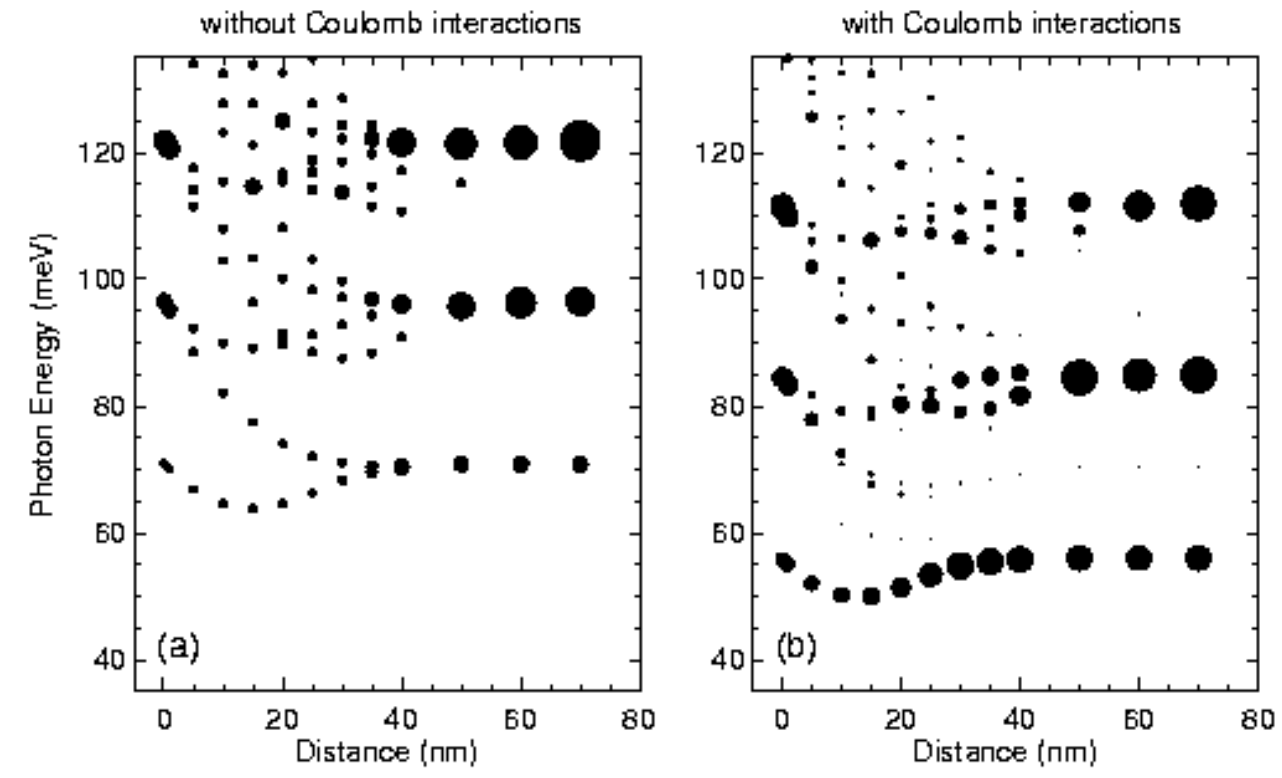

FIG. 3. Same as Fig. 2; the size of each dot corresponds to the height (i.e., oscillator strength) of the corresponding absorption peak. 


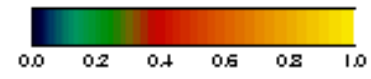

without Coubmb interactions

with Coubmb interactions
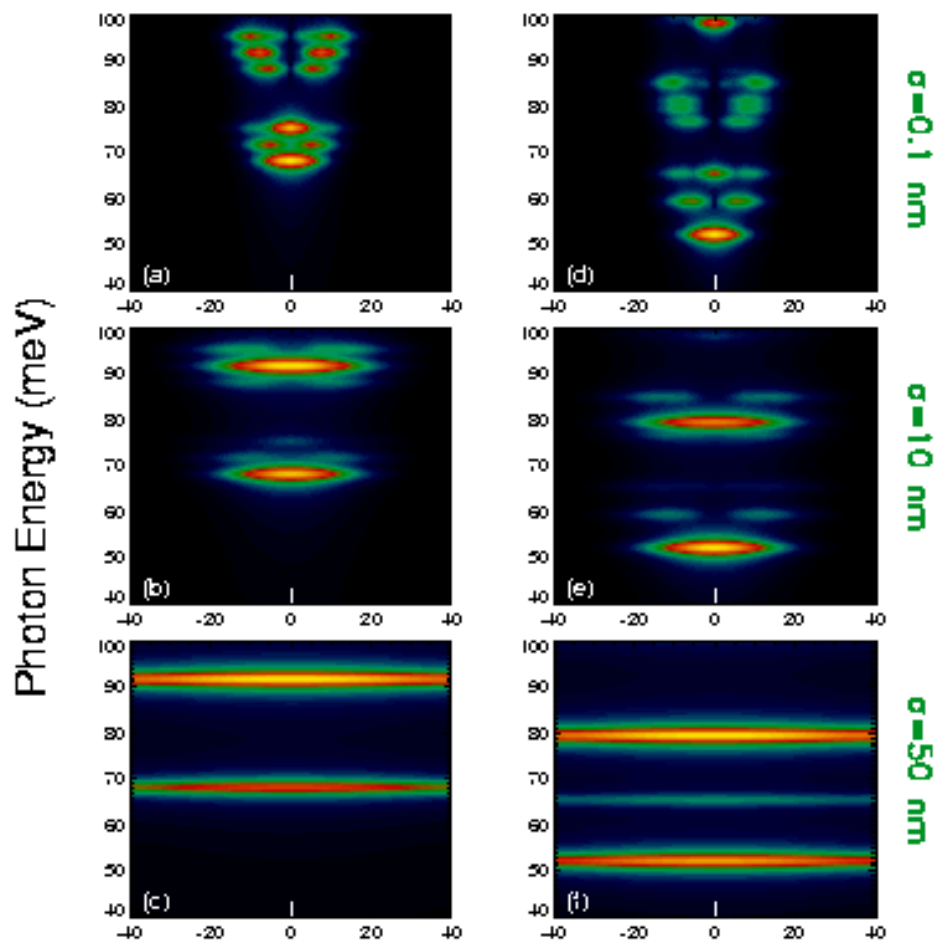

Position (nm)

FIG. 4. Local absorption spectra $\alpha_{\xi}(X, \hbar \omega)$ for a single QD with (Figs. $4(\mathrm{~d}-\mathrm{f})$ ) and without (Figs. 4(a-c)) Coulomb interactions and for different values of $\sigma$. Photon energy $\hbar \omega$ is measured with respect to the bandgap, and $X$ is the position of the tip along the $x$-axis $(Y=0)$. In this calculations we use a basis of 6 electron and hole states, respectively. 


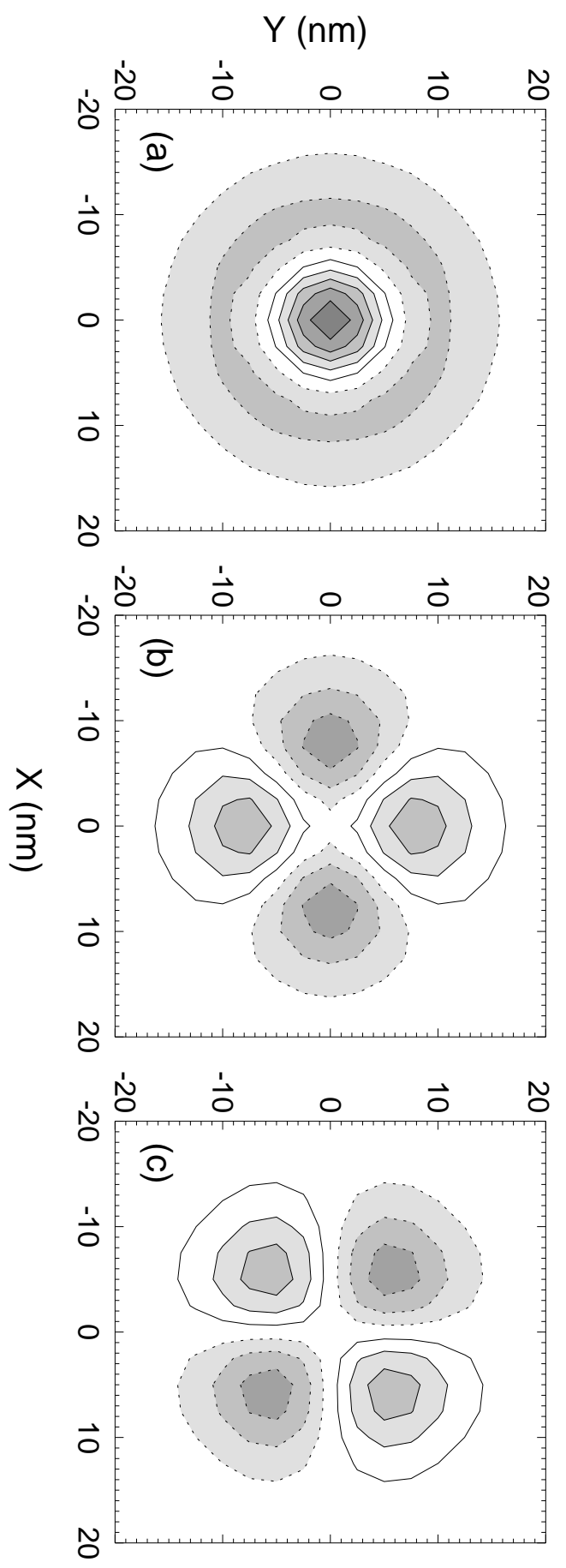

FIG. 5. Contour plot of the exciton wavefunction $\Psi^{\lambda}(\mathbf{r}, \mathbf{r})$ for three excitons which contribute to the absorption peak at $\approx 65$ $\mathrm{meV}$. Solid and dashed lines correspond to positive and negative values, respectively. 


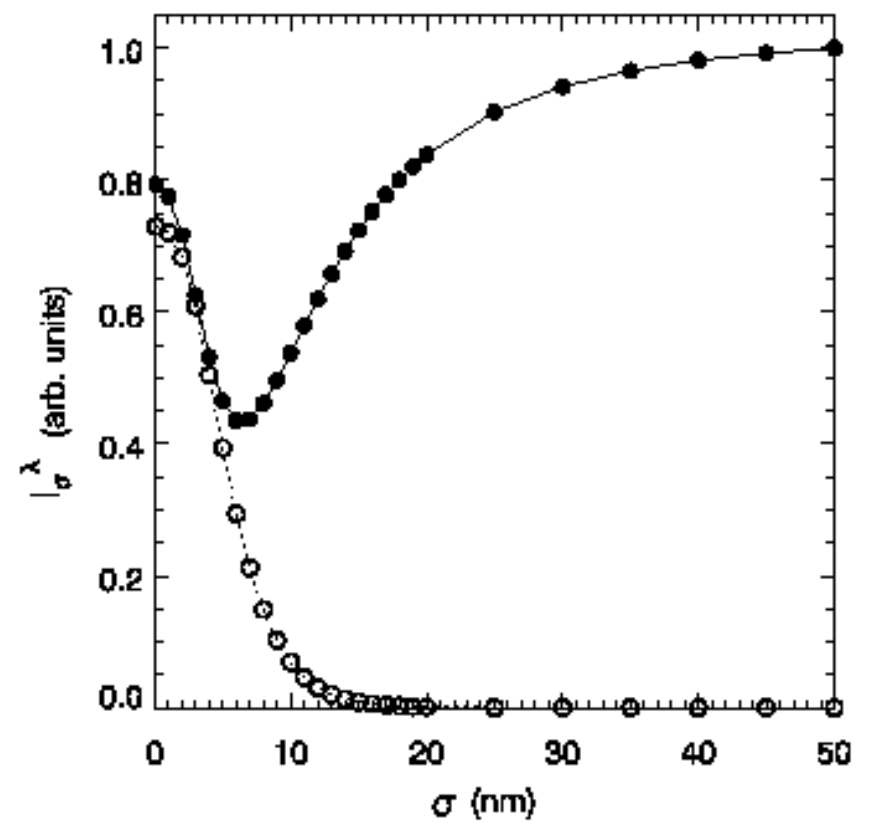

FIG. 6. The relative contribution, $I_{\xi}^{\lambda}$, as a function of $\sigma$, for the excitons (depicted in Fig.5) which are responsible for the non-monotonic behavior of the feature at $65 \mathrm{meV}$. 
Photon Energy (meV)
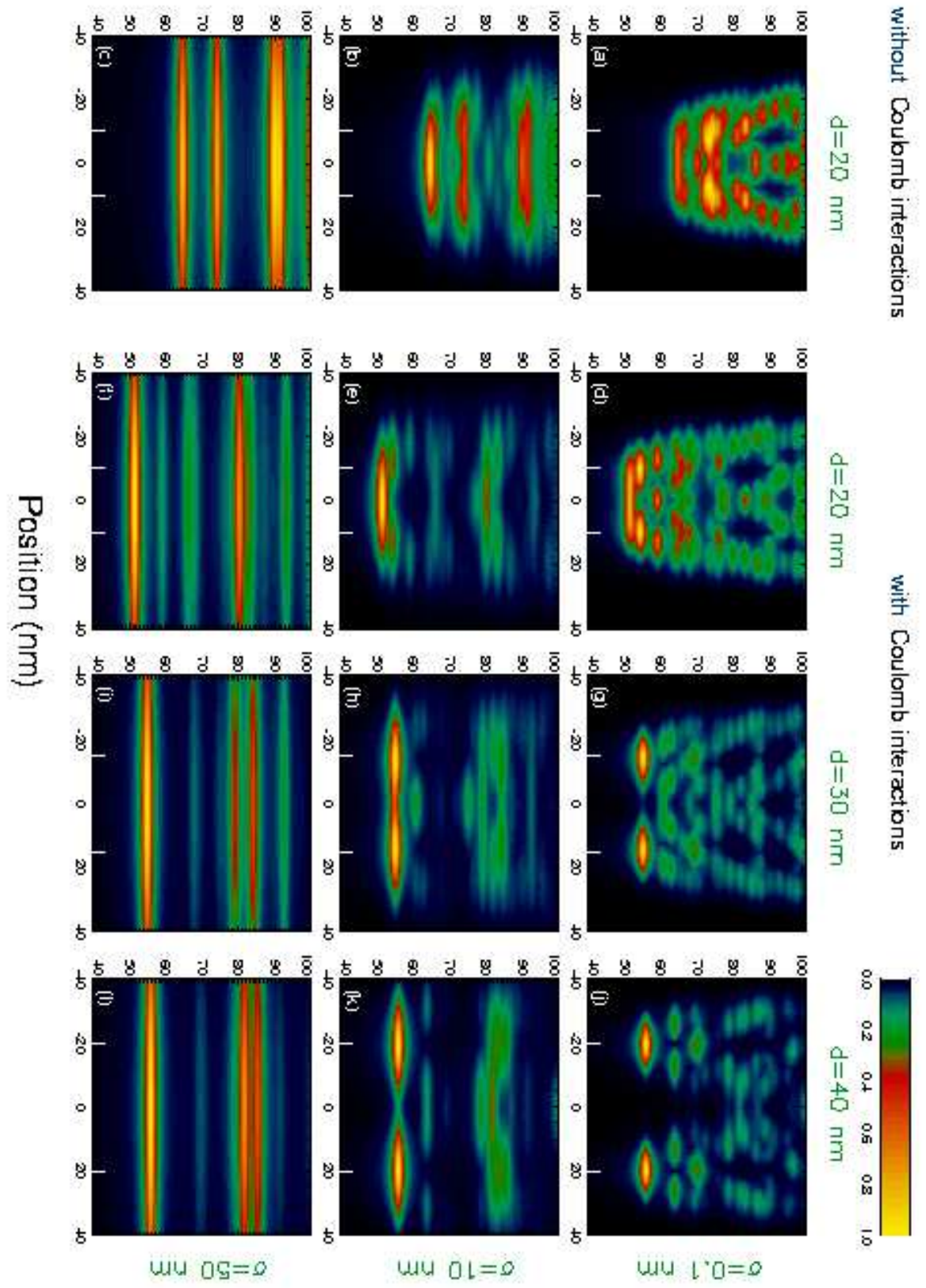

FIG. 7. Local absorption spectra $\alpha_{\xi}(X, \hbar \omega)$ for a double QD with (Figs. 7(d-l)) and without (Figs. 7(a-c)) Coulomb interactions and for different values of $\sigma$ and interdot distance $d$. Photon energy $\hbar \omega$ is measured with respect to the bandgap, and $X$ is the position of the tip along the $x$-axis $(Y=0)$. In our calculations we use a basis of 12 electron and hole states, respectively. 

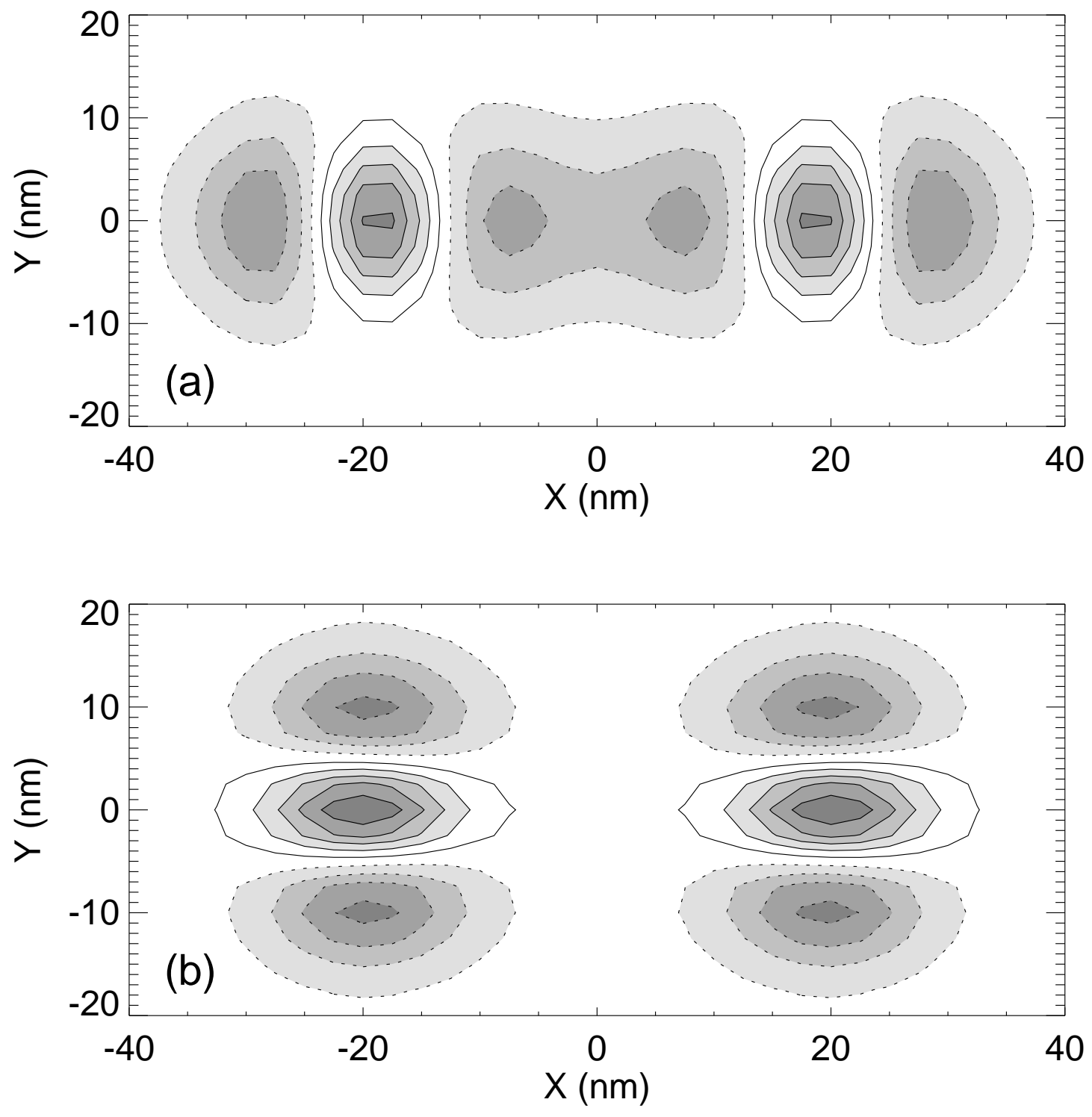

FIG. 8. Contour plot of the exciton wavefunction $\Psi^{\lambda}(\mathbf{r}, \mathbf{r})$ of the two excitons which are responsible for the non-monotonic behaviour of the features at $\approx 70 \mathrm{meV}$ at the interdot distance $d=40 \mathrm{~nm}$. Solid and dashed lines correspond to positive and negative values, respectively. The upper (lower) panel refers to exciton with energy $69.1 \mathrm{meV}(70.3 \mathrm{meV})$. 